Cahiers de recherches médiévales

La réception d'Isidore de Séville durant le Moyen Âge tardif $\left(\left.X I\right|^{e}-X V^{e}\right.$ s.)

\title{
Les .XV. Joyes nostre Dame rimees de Christine de Pizan
}

Jean-François Kosta-Théfaine

\section{CpenEdition}

\section{Journals}

Édition électronique

URL : https://journals.openedition.org/crm/10982

DOI : $10.4000 / \mathrm{crm} .10982$

ISSN : 1955-2424

Éditeur

Honoré Champion

Édition imprimée

Date de publication : 10 décembre 2008

Pagination : 255-277

ISSN : 1272-9752

Référence électronique

Jean-François Kosta-Théfaine, "Les .XV. Joyes nostre Dame rimees de Christine de Pizan », Cahiers de recherches médiévales [En ligne], 16 | 2008, mis en ligne le 15 décembre 2011, consulté le 15 décembre 2022. URL : http://journals.openedition.org/crm/10982 ; DOI : https://doi.org/10.4000/crm.10982 


\section{RM}

\section{Les .XV. Joyes nostre Dame rimees de Christine de Pizan ${ }^{1}$}

L'œuvre de Christine de Pizan est importante tant d'un point de vue qualitatif que quantitatif. L'attention portée à celle-ci est, sans excès, conséquente et il faut souligner qu'un grand nombre d'études lui a été consacré ${ }^{2}$. Cet intérêt n'a toutefois pas pris en considération certains textes. En effet, cela est vrai s'agissant, parmi quelques autres, de ses compositions religieuses qui, à ce jour, n'ont été l'objet que de peu d'investigations ${ }^{3}$. On peut supposer que cela est dû au simple fait qu'elles ont

\footnotetext{
${ }^{1}$ Nous tenons à remercier L. Dulac, Ch. Reno et E.J. Richards pour leur précieux conseils.

${ }^{2}$ En témoignent les deux guides bibliographiques de A. J. Kennedy, Christine de Pizan : A Bibliographical Guide, Londres, (Research Bibliographies and Checklists, 42), Grant \& Cutler Ltd., 1984 ; A. J. Kennedy, Christine de Pizan : A Bibliographical Guide. Supplement I, Londres, (Research Bibliographies and Checklists, 42.1), Grant \& Cutler Ltd., 1994 et A. J. Kennedy, Christine de Pizan: A Bibliographical Guide. Supplement II, Woodbridge, (Research Bibliographies and Checklists: New Series 5), Tamesis, 2004. Voir également E. Yenal, Christine de Pizan : a bibliography, Metuchen, N. J. et Londres, (Scarecrow Author Bibliography, 63), The Scarecrow Press, 1989 ; A. J. Kennedy, «A Selective Biblography of Christine de Pizan Scholarship, circa 1980-1987», in Reinterpreting Christine de Pizan, éd. E. J. Richards with J. Willamson, N. Margolis and Ch. Reno, Athens \& London, The University of Georgia Press, 1992, p. 285-298 et J.-F. Kosta-Théfaine, «Bibliographie du Ditié de Jehanne d'Arc (1429) de Christine de Pizan », Ariane, 16, 1999-2000, p. 213-221.

${ }^{3}$ Les études qui leur ont été consacrées sont les suivantes: S. Solente, Christine de Pizan, Paris, Éditions Klincksieck et Imprimerie Nationale, 1969, (p. 24, 63-64, 74); L. Deslile, «Notice sur Les Sept Psaumes Allegorisés de Christine de Pizan», Notices et extraits des manuscrits de la Bibliothèque Nationale et autres bibliothèques, XXXV, 2ème partie, 1896, p. 551-559; L. Dulac, «Un écrit militant de Christine de Pizan : le Ditié de Jehanne d'Arc », in Aspects of Female Existence-Proceedings from the St. Gertrud Symposium "Women in the Middle Ages », Copenhagen, September 1978, éd. B. Carlé, N. Damhsholt, K. Glente et E. T. Nielsen, Danemark, Glydendal, 1980, p. 115-134, (p. 116-117); S. Solente, «Un traité inédit de Christine de Pizan : l'Epistre de la Prison de Vie Humaine », Bibliothèque de l'École des Chartes, LXXXV, 1924, p. 263-301, (p. 326); G. Gros, «mon oroison entens... Étude sur trois opuscules pieux de Christine de Pizan », Bien dire et bien aprandre, 8, 1990, p. 99-112 ; G. Wall, "Christine de Pizan: The .XV. Joys of our Lady», Vox Benedictina, 2, 1985, p. 134-147 ; N. Margolis, «La progression polémique, spirituelle et personnelle dans les écrits religieux de Christine de Pizan ", in Une femme de lettres au Moyen Âge. Études autour de Christine de Pizan, éd. L. Dulac et B. Ribémont, Orléans, Paradigme, 1995, p. 297-306 ; Ch. C. Willard, "Christine de Pizan's Allegorized Psalms", in Une femme de lettres au Moyen Âge. Études autour de Christine de Pizan, éd. L. Dulac et B. Ribémont, Orléans, Paradigme, 1995, p. 317-324 ; L. Walters, «The Royal Vernacular: Poet and Patron in Christine de Pizan's Charles $V$ and the Sept Psaumes Allegorisés, The Vernacular Spirit: Essays on Medieval Religious Literature, éd. R. Blumenfeld-Kosisnki, D. Robertson, \& N. Bradley
}

Cahiers de Recherches Médiévales, 16, 2008 
toujours été considérées comme des textes qui ne constituent pas, dans l'ensemble de sa production, un élément significatif de celle-ci. Elles ne sont pourtant pas dénuées d'une certaine importance dans la carrière littéraire de Christine de Pizan et méritent un intérêt à la hauteur de leur valeur et de leur qualité.

Il convient de rappeler que Christine de Pizan a rédigé plusieurs textes religieux, en plus de la rédaction de trente et un textes qui s'apparentent tant au traité didactique que politique, auxquels s'ajoute la composition de trois cent soixante quatorze pièces lyriques, dont une partie se trouve insérée dans certains de ses dits narratifs. ${ }^{4}$. Elle a, en effet, composé trois opuscules pieux: L'Oroyson nostre Seigneur ${ }^{5}, L^{\prime}$ Oroyson nostre Dame ${ }^{6}$ et Les .XV. Joyes nostre Dame rimees ${ }^{7}$; un service d'Heures : Les Heures de Contemplacion sur la Passion de nostre Seigneur ${ }^{8}$

Warren, New York, Palgrave, 2002, p. 145-182 ; G. Gros, Le Poète, la Vierge et le Prince. Étude sur la poésie mariale en milieu de cour aux XIV et XV siècles, Saint-Étienne, Publications de l'Université de Saint-Étienne, 1994, p. 79-92; J.-F. Kosta-Théfaine, «Le Ditié de Jehanne d'Arc de Christine de Pizan : un modèle d'architecture fondé sur l'utilisation de la symbolique des Nombres », Cahiers de Recherches Médiévales, 3, 1997, p. 121-129, (p. 124-125) et M. Boulton, «Nous deffendons de feu, ... de pestilence, de guerre: Christine de Pizan's Religious Works », in Christine de Pizan. A Casebook, ed. B. K. Altmann and D. L. McGrady, London and New York, Routledge, 2003, p. 215-228.

${ }^{4}$ Il s'agit des textes suivants : Dit de la Rose, in CEuvres poétiques de Christine de Pizan, éd. M. Roy, Paris, Firmin Didot, tome II, 1896-Rééd., New York, Johnson Reprints, 1965, p. 2948, (qui regroupe trois ballades et un rondeau); Dit de la Pastoure, in Oeuvres poétiques de Christine de Pizan, op. cit., tome II, p. 223-294, (où sont insérés trois bergerettes, quatre ballades et un rondeau) et Livre du Duc des Vrais Amans, in Euvres poétiques de Christine de Pizan, op. cit., tome III, p. 59-208 et Christine de Pizan, Livre du Duc des Vrais Amans, éd. Th. Fenster, New York, Medieval \& Renaissance Texts \& Studies, 1995, (qui contient sept rondeaux, vingt quatre ballades, quatre virelais et une complainte). Nous trouvons également une ballade insérée dans L'Avision-Christine, éd. M. L. Towner, Washington D.C., The Catholic University of America Press, 1932-Rééd., New York, AMS Press, 1965, p. 159160 (Le Livre de l'advision Cristine, éd. Ch. Reno et L. Dulac, Paris, Champion, 2001, p. 105-106) et un rondeau dans l'Epistre a la Reine, éd. A.J. Kennedy, Revue des Langues Romanes, 92:2, 1988, p. 253-264, (p. 258). Voir également J.-F. Kosta-Théfaine, «Les Virelais de Christine de Pizan », Le Moyen Français, 47, 2001, p. 123-145.

${ }^{5}$ Christine de Pizan, Oroyson Nostre Seigneur, in Euvres poétiques, op. cit., tome III, p. 1526.

${ }^{6}$ Christine de Pizan, Oroyson Nostre Dame, in Euvres poétiques, op. cit., p. 1-9. Cf. également : in R. Thomassy, Essai sur les écrits politiques de Christine de Pizan, suivi d'une notice littéraire et de pièces inédites, Paris, Debécourt, 1838, p. 171-181 ; Oroyson Nostre Dame, Utrecht, De Roos, 1948 et Oroyson Nostre Dame : Prayer to Our Lady by Christine de Pizan, tr. J. Misrahi et M. Marks, New York, Kurt H. Volk, 1953.

${ }^{7}$ Christine de Pizan, .XV. Joyes nostre Dame, in Euvres poétiques, op. cit., p. 11-14.

${ }^{8}$ Ce service d'Heures nous a été conservé dans les deux manuscrits suivants : le ms. Paris, B.N. nouv. acq. fr. 10059 (ff. 114r-144r) et le ms. La Haye, Bibliothèque Royale, 73. J. 55 (ff. 51r-92v). L. Dulac prépare actuellement une édition critique de ce texte. Nous la remercions de nous en avoir communiqué une version provisoire. Sur ce texte, on consultera avec profit l'étude de L. Dulac, "Littérature et dévotion: À propos des Heures de 
et une traduction des psaumes pénitentiaux qui, selon la numérotation de la Vulgate, sont les psaumes 6, 31, 37, 50, 101, 129 et 120, intitulée Les Sept Psaumes allégorisés $^{10}$. De fait, si l'on porte une attention particulière à son œuvre religieuse, il est possible de distinguer trois périodes distinctes la concernant. La première (14021403) est constituée par la composition des trois opuscules pieux susmentionnés ; la deuxième (vers 1409) par la rédaction des Sept Psaumes allégorisés et enfin une troisième (entre 1418 et 1429) qui correspond à l'écriture des Heures de Contemplacion. De même, ces trois périodes ainsi distinguées permettent de mettre en évidence, comme l'a fait observer Nadia Margolis ${ }^{11}$, une progression polémique, spirituelle et personnelle chez cet auteur, au sens où il s'agit, pour elle, de délivrer un message qui se veut à la fois politique, moral et féministe.

\section{Le texte}

Les .XV. Joyes nostre Dame rimees sont constitutives, dans l'œuvre de Christine Pizan, des débuts de sa carrière littéraire. Cette dernière, tout en étant à ses prémices aux alentours des années 1402-1405, n'en est pas moins importante. En effet, rappelons qu'avant cette période, elle a déjà écrit quelque neuf textes. Il faut ajouter, de plus, que la composition de son œuvre lyrique s'échelonne entre 1393 et 1410. Christine de Pizan compose cette prière à un moment où elle commence à diversifier le genre de ses écrits ${ }^{12}$, avec des textes tels que le Livre du Chemin de Long Estude (1402-1403), le Livre de la Mutacion de Fortune (1403), le Livre des fais et bonnes meurs su sage roy Charles V (1404), le Livre de la Cité des Dames (1405) et d'autres encore.

Cette pièce religieuse s'inscrit, en outre, dans un mouvement caractéristique des $\mathrm{XIV}^{\mathrm{e}}$ et $\mathrm{XV}^{\mathrm{e}}$ siècles qui voit éclore la poésie mariale ${ }^{13}$. Cette dernière sera, du reste, favorisée par Charles VI qui, selon l'expression d'Hélène Millet, «probable

contemplacion sur la Passion de Nostre Seigneur de Christine de Pizan», Miscellanea mediaevalia. Mélanges offerts à Philippe Ménard, études réunies par J.-C. Faucon, A. Labbé et D. Quéruel, Paris, Champion, 1998, tome I, p. 475-484.

${ }^{9}$ Voir à ce propos Ch. C. Willard, «Christine de Pizan's Allegorized Psalms », op. cit., p. 317-318.

${ }^{10}$ Christine de Pizan, Sept Psaumes allégorisés, éd. R. R. Rains, Washington D.C., The Catholic University of America Press, 1965; B. Ribémont prépare une nouvelle édition de ce texte.

${ }^{11}$ Voir à ce sujet N. Margolis, «La progression polémique, spirituelle et personnelle dans les écrits religieux de Christine de Pizan », op. cit., p. 298.

${ }^{12} \mathrm{Ch}$. C. Willard a également souligné, pour sa part, que «by 1402 Christine was turning her attention to more serious forms of writting, although she continued to compose love poetry for several years ", in Ch. C. Willard, Christine de Pizan, Her Life and Works, op. cit., p. 54.

${ }^{13}$ Voir à ce propos G. Gros, Le Poète, la Vierge et le Prince, op. cit. à la note 4. Sur la dévotion mariale, on consultera, entre autres, les ouvrages suivants: A. Vauchez et Ch. Vincent, Dictionnaire encyclopédique du Moyen Âge, Paris, Éditions du Cerf, 1997 : voir l'entrée «Marié », MARIA. Études sur la Sainte Vierge, sous la direction de H. Du Manoir, Paris, Beauchesne, 1949-1964, B. Béthouard et A. Lottin, La dévotion mariale de l'an mil à nos jours, Arras, Artois Presses Université, 2005 et D. Iogna-Prat, Le culte de la Vierge dans la société médiévale, Paris, Beauchesne, 1996. 
destinataire de cette poésie, avait hérité de son père une solide piété $»^{14}$. L'Hôtel royal était, comme l'a montré Xavier de La Selle, un lieu propice à la religion ${ }^{15}$. Ainsi, nombreux étaient les poètes à composer des pièces dévotes, parmi lesquels, outre Christine de Pizan, figuraient Guillaume de Machaut ou bien encore Eustache Deschamps, pour ne citer qu'eux. Mais de l'avis d'Hélène Millet, «il n'y a guère que Christine de Pizan à avoir composé suffisamment de vers pieux pour être mise en balance avec Eustache Deschamps $\gg{ }^{16}$.

Lorsque Christine de Pizan rédige Les. $X V$. Joyes nostre Dame rimees, il semble qu'elle tente de composer une prière ayant une tonalité pleinement personnelle. Elle s'éloigne alors du dessein qui était le sien dans L'Oroyson nostre Dame, dont la visée paraît beaucoup plus universelle, puisqu'elle concerne la France. Cette dernière se trouve, en effet, ordonnée selon la théorie des états du monde. On peut alors observer tout au long de cette pièce, comme l'a montré Gérard Gros, qu'une série de demandes se trouve formulée selon une hiérarchie sociale bien précise $^{17}$ :

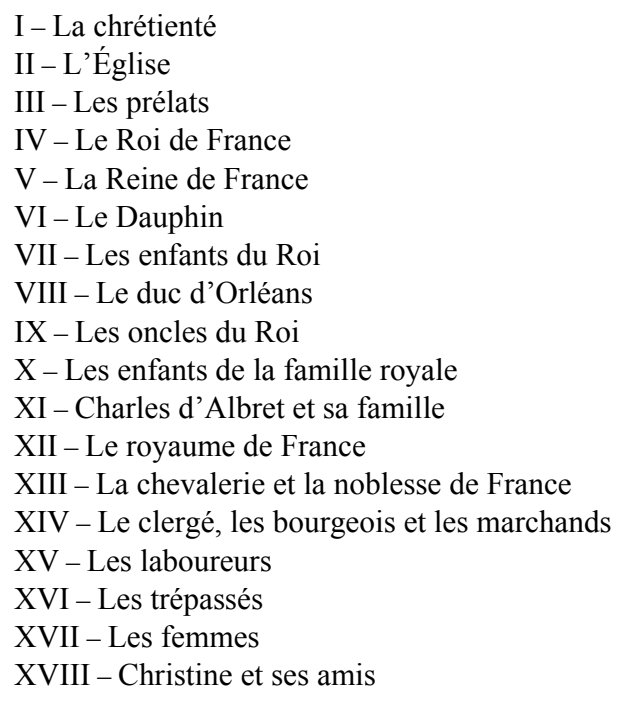

\footnotetext{
${ }^{14}$ H. Millet, «Expression d'une foi », in Eustache Deschamps en son temps, sous la direction de J.-P. Boudet et H. Millet, Paris, Publications de la Sorbonne, 1997, p. 63-87, (p. 77).

${ }^{15} \mathrm{X}$. de la Selle, Le service des âmes à la cour. Confesseurs et aumôniers des rois de France du XIII au XV siècles, Paris, École des Chartes, 1995.

${ }^{16}$ H. Millet, «Expression d'une foi », in Eustache Deschamps en son temps, op. cit., p. 77.

${ }^{17}$ Voir G. Gros, «mon oroisons entens... Étude sur les trois opuscules pieux de Christine de Pizan », op. cit., p. 103 et G. Gros, Le Poète, la Vierge et le Prince. Étude sur la poésie mariale en milieu de cour aux XIV et XV siècles, op. cit., (p. 79-92), p. 84.
} 
Christine semble, notons-le, procéder d'une manière quelque peu similaire dans le Ditié de Jehanne d'Arc ${ }^{18}$. Elle y multiplie, en effet, les destinataires. Ainsi, selon les termes de Liliane Dulac, «après avoir convié l'ensemble des Français 'grant et menu'-public qu'elle apostrophe à d'autres moments du poème- à se réjouir avec elle, Christine s'adresse :

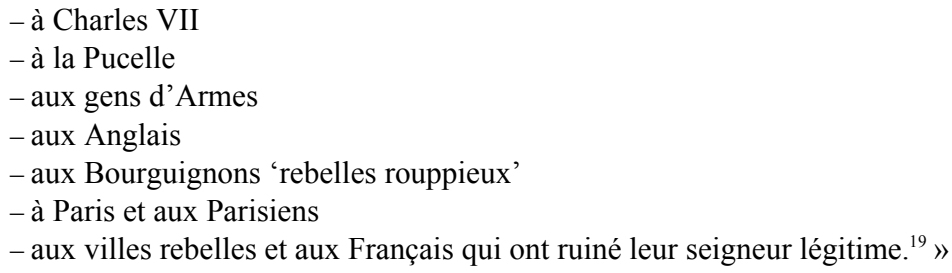

La facture de L'Oroyson nostre Dame apparaît donc comme étant assez moraliste. Il faut toutefois souligner que la moraliste Christine qui se fait jour à travers cette oraison ne tente à aucun moment de dénoncer quoi que ce soit. De fait, comme l'a très justement écrit Gérard Gros, «les faiblesses [que la poétesse] constate se convertissent en motifs d'une prière qu'elle supplie la Vierge de faire exaucer. En retour, le propos de Christine montre implicitement aux dédicataires princiers, ses lecteurs éventuels, comment on peut détenir le pouvoir et rester humble $»^{20}$.

La prière des . $X V$. Joyes nostre Dame rimees s'inscrit essentiellement dans le sillage d'une paraphrase, de la manière la plus simple et la plus directe qui soit, des sources religieuses. Sa composition est, en outre, relativement simple puisqu'elle comprend seize quatrains d'octosyllabes à rimes croisées, avec l'ajout, après chacune des strophes, de l'Ave Maria. Il est à noter qu'à partir du XII ${ }^{\mathrm{e}}$ siècle on aime associer la répétition «Ave Maria » au rappel des « Cinq Joies de la Vierge » ${ }^{21}$. La structure de la prière de Christine de Pizan pourrait même faire penser que cette prière a été composée avec l'objectif d'être récitée à haute voix. Cette hypothèse laisserait alors supposer que ce poème religieux s'apparente à une prière composée par une laïque respectant dévotement l'Église. Une autre interprétation est, vraisemblablement, envisageable. Ainsi, pour Hélène Millet, «Christine semble avoir voulu conférer un statut décoratif et secondaire tout à fait similaire $»^{22}$ à ces somptueuses décorations qui ornent bon nombre de livres d'Heures et dont l'objectif était alors d'inviter à la méditation de la contemplation par la beauté. Cela permettait alors de compenser, selon son expression, «la pauvreté de la participation de

\footnotetext{
${ }^{18}$ Christine de Pizan, Ditié de Jehanne d'Arc, éd. A. J. Kennedy et K. Varty, Oxford, Medium Aevum Monographs, (New Series, IX), 1977.

${ }^{19}$ L. Dulac, «Un écrit militant de Christine de Pizan : le Ditié de Jehanne d'Arc », op. cit., p. 127.

${ }^{20}$ G. Gros, «mon oroison entens... Étude sur trois opuscules pieux de Christine de Pizan », op. cit., p. 103-104.

${ }^{21}$ Sur Les Joies de Marie, voir l'histoire du Rosaire. Nous tenons à remercier Liliane Dulac de nous en avoir communiqué un exemplaire.

${ }^{22}$ H. Millet, «Expression d'une foi », in Eustache Deschamps en son temps, op. cit., p. 77.
} 
l'intellect aux exercices spirituels $\rangle^{23}$. Enfin, une troisième hypothèse vient d'être récemment émise par Maureen Boulton. Cette dernière voit, en effet, à travers l'utilisation du syntagme "Ave Maria», "an imitation of the treatment of the invitatory (Psalm 94), where pairs of psalm verses are punctuated with the beginning of the Ave Maria $»^{24}$. De plus, toujours d'après Maureen Boulton, Christine de Pizan se serait inspirée, pour la composition de ces .XV. Joyes nostre Dame rimees, de la prière en prose intitulée Doulce Dame de misericorde qui, communément copiée dans les livres d'Heures, se trouve également ponctuée par un «Ave Maria gratia plena » après chacune des joies. Dès lors, selon les termes de Maureen Boulton, « in versifying the prayer, Christine produced a poem of extraordinary concision and stylistic variation, and gave the intrusive Latin prayer a formal function in her poem by using it as a refrain for each stanza $»^{25}$.

À travers cette pièce, que Gérard Gros qualifie, à juste titre, de «prière éminemment féminine $»^{26}$, émerge l'idée d'une exaltation de la maternité. Cette dernière est suggérée par le biais de la célébration de la Vierge qui est, avant toute chose, une femme à part entière. Elle est celle qui, tout comme l'auteur de ces Joies, a vécu les mêmes douleurs : celle de l'enfantement mais aussi et surtout, celle de la perte du fruit de ses entrailles ${ }^{27}$. En outre, si Marie apparaît de manière certaine comme étant le garant du salut de l'être humain, on prend rapidement conscience que sous la plume de Christine de Pizan, les hommes comptent, beaucoup plus que les femmes, sur la Vierge pour les sauver. La raison en est simple: «c'est aux femmes, reflet terrestre de la Vierge, [qu'il incombe] de sauver le monde $»^{28}$.

Le fait de composer ce type de prière peut constituer, de la part de Christine, une certaine forme d'originalitée ${ }^{29}$, car si les Quinze joies de Marie en vers ou en prose existaient depuis longtemps, ces textes étaient en latin. De plus, si l'on

\footnotetext{
${ }^{23}$ H. Millet, « Expression d'une foi », in Eustache Deschamps en son temps, op. cit., p. 77.

${ }^{24} \mathrm{M}$. Bouton, «Nous deffendons de feu, ... de pestilence, de guerre: Christine de Pizan's Religious Works », in Christine de Pizan : A Casebook, op. cit., p. 213.

${ }^{25} I d .$, p. 219.

${ }^{26}$ G.Gros, «mon oroison entens... Étude sur trois opuscules pieux de Christine de Pizan », op. cit., p. 106. Il est à noter que E. Bayer dans un artcile du Marienlexikon corrobore cette hypothèse de G. Gros, voir E. Bayer, «Sieben Freuden Marien », Marienlexikon, St. Otillien, Eos Verlag, 1994, v. 6, p. 155.

${ }^{27}$ Rappelons que Christine de Pizan a perdu l'un de ses fils avant 1400. Charity Cannon Willard écrit, à ce propos, que «Little is known about Christine's third child, a son. He must have died around the same time the other two children left Christine's roof, for when Jean left for England she spoke of him as the older of her two sons, but when he returned home three years later, she said that death had left her only one son. ", in Ch. C. Willard, Christine de Pizan: Her Life and Works, op. cit., p. 43.

${ }^{28}$ Nous empruntons cette phrase à N. Margolis, «La progression polémique, spirituelle et personnelle dans les écrits religieux de Christine de Pizan », op. cit., p. 300.

${ }_{29}$ Notons qu'il existe une prière en prose extraite d'un manuscrit de la première moitié du $\mathrm{XV}^{\mathrm{e}}$ siècle dont l'extrême ressemblance avec celle en vers de Christine de Pizan est assez frappante. Voir H. Suchier, "Les quinze joies nostre dame», Zeitschrift für Romanische Philologie, 17: 1-2, 1893, p. 282-285. Nous remercions Liliane Dulac de nous avoir signalé l'existence de ce texte et de nous en avoir communiqué une copie.
} 
rencontre fréquemment des cinq, sept, neuf ou bien encore douze « Joies » aux XII ${ }^{\mathrm{e}}$ et $\mathrm{XIII}^{\mathrm{e}}$ siècles $^{30}$, il faut souligner, avec Gérard Gros ${ }^{31}$, qu'en ce qui concerne la forme des «XV Joies », il n'en existe que deux exemples pour ce qui est du Moyen Âge tardif ${ }^{32}$ :

- «Recorder voil la joie/Ke veroyment esteit riviere» par Martin, moine de Bury (15 strophes d'Hélinant et un corollaire de 6 vers) ${ }^{33}$.

- «Vierge, qui par ta digneté/Par ta sainte humilité» (519 octosyllabes à rimes suivies $)^{34}$.

Christine de Pizan fait, en revanche, moins preuve d'originalité à travers le motif qu'elle développe ici, même si l'on observe toutefois que ces .XV. Joyes nostre Dame rimees trouvent leur correspondance dans L'Oroyson nostre Seigneur ${ }^{35}$. Il faut souligner, par ailleurs, que les .XV. Joyes nostre Dame rimees et l'Oroyson nostre Seigneur qui ne sont que des prières historiées dont les sources sont incontestablement les Écritures, se trouvent composées selon un entrelacement thématique facilement repérable:

\footnotetext{
${ }^{30}$ Notons qu'un grand nombre de prières, dont beaucoup sont encore inédites, nous ont été transmises, comme l'on sait, par le biais de livres de prières, de livres d'Heures ou bien encore de recueils de textes dévots. Ces pièces pieuses font l'objet, depuis plusieurs années maintenant, d'un recensement systématique : voir J. Sonnet, Répertoire d'incipit de prières en ancien français, Genève, Droz, 1956 ; P. Rezeau, Répertoire des prières françaises à la fin du Moyen Âge, Genève, Droz, 1986; K. V. Sinclair, Prières en ancien français, HandenConnecticut, Archon Books, 1978; K. V. Sinclair, French Devotional Texts of the Middle Ages, Wesport-Connecticut et Londres, Greenwood Press, 1979; K. V. Sinclair, French Devotional Texts of the Middle Ages-1st Supplement, Wesport-Connecticut et Londres, Greenwood Press, 1982 et K. V. Sinclair, French Devotional Texts of the Middle Ages-2nd Supplement, Wesport-Connecticut et Londres, Greenwood Press, 1988.

${ }^{31} \mathrm{G}$. Gros, «mon oroison entens... Étude sur trois opuscules pieux de Christine de Pizan », op. cit., p. 105.

${ }^{32}$ Sur la spiritualité au Moyen Âge, voir entre autres : N. Bériou, T. Berlioz et J. Longère (éd.), Prier au Moyen Âge. Pratiques et expériences religieuses, Turnhout, Brepols, 1991 ; B. Médrignac, La Vie religieuse en France au Moyen Âge, Paris, Ophrys, 1994 et A. Vauchez, La Spiritualité du Moyen Âge en occident (VIII - -XIII ${ }^{e}$ siècles), Paris, Le Seuil, 1994.

${ }^{33} \mathrm{~J}$. Sonnet, Répertoire d'incipit de prières en Ancien Français, op. cit., $\mathrm{n}^{\circ} 1768$.

${ }^{34} I d ., \mathrm{n}^{\circ} 2331$.

${ }^{35}$ Notons que cette dernière, écho à l'Oroyson nostre Dame, ne fait que reprendre le motif de la Passion.
} 
.XV. Joyes nostre Dame rimees

Annonciation

Nativité

Visite des bergers

Oblation des Rois

Offrande au temple

Apparition du ressuscité

Ascension

Pentecôte
I

IV

V

VI

VII

XII

XIII

XIV
Oroyson nostre Seigneur

III

IV

V

VII

VIII

LVII

LIX

LX

Les .XV. Joyes nostre Dame rimees, tout comme l'ensemble de l'œuvre religieuse de Christine de Pizan, révèlent une autre facette, mal connue, de cet écrivain. Ces compositions pieuses méritent une attention certaine et un autre intérêt que celui, infime, que les exégètes ont bien voulu leur accorder à ce jour.

\section{Édition et traduction des .XV. Joyes nostre Dame rimees}

La première et unique édition des .XV. Joyes nostre Dame rimees a été établie par Maurice Roy, dans le troisième volume des Euvres poétiques de Christine de Pizan, sous le titre: .XV. Joyes nostre Dame. Les trois volumes de ces Euvres poétiques, publiés à Paris, entre 1886 et 1896, par Firmin-Didot, ont été fort utiles et ont rendu un grand service, car ils ont permis de mettre à la disposition de tout un chacun l'ensemble des œuvres poétiques de Christine de Pizan. Malgré cela, il faut reconnaître qu'ils sont à la fois fort anciens et, malgré une réédition à New York par Johnson Reprints en 1965 - elle-même épuisée à ce jour -, d'un accès peu facile. De plus, tous les manuscrits contenant le texte des .XV. Joyes nostre Dame rimees ne sont pas pris en considération. En effet, Maurice Roy n'a utilisé que quatre manuscrits sur les cinq qui ont conservé cette pièce, à savoir les manuscrits Paris, BnF fr. $836(A 1)$, Londres, British Library, Harley 4431 (A2), Paris, BnF fr. 12779 (B2) et Chantilly, Musée Condé 492 (B3). Or, ce texte se trouve dans un cinquième manuscrit: Paris, Arsenal 3295 (B4) qui doit, comme c'est le cas dans notre édition, être pris en considération. En outre, Maurice Roy a établi le texte de cette prière d'après le ms. Paris, $\mathrm{BnF}$ fr. 836, alors que les recherches de ces dernières années ont clairement mis en évidence qu'il était préférable d'utiliser comme manuscrit de base le ms. Londres, B. L., Harley 4431. Enfin, l'édition de Maurice Roy n'était accompagnée ni de notes ni de glossaire. Une nouvelle édition de ce texte était donc à envisager.

Il existe une traduction anglaise de cette prière. Elle a été établie par Glenda Wall et a été publiée dans la revue Vox Benedictina en 1985. Cette traduction est précédée d'une introduction portant sur la vie de Christine, la place de cette prière 
par rapport à son œuvre ainsi que quelques réflexions sur ce texte. Elle n'est pas accompagnée de notes.

\section{Les manuscrits}

La prière des.$X V$. Joyes nostre Dame rimees nous a été conservée dans les cinq manuscrits suivants ${ }^{36}$ :

- ms. Paris, BnF fr. 836 (ff. 47r-48r) : A1.

Manuscrit (anc. 7216) en parchemin mesurant $352 \times 260 \mathrm{~mm}$ et composé de 98 feuillets, écrit sur deux colonnes. Ce manuscrit fait partie d'un ensemble de cinq manuscrits $(\mathrm{BnF}$ fr. 835, 836, 605, 606 et 607) qui renferment les Euvres de Christine de Pizan. Il est généralement désigné par l'appellation «Livre du Duc $»^{37}$. Il aurait, en effet, probablement d'abord été destiné au duc d'Orléans, mais c'est en fait le duc de Berry qui en fit l'acquisition en 1408-1409. Il renferme les textes suivants : Livre du chemin de long estude (ff. 1r-41v); Enseignemens moraux (42r45v); Oroyson Nostre Dame (ff. 45r-47r) ; .XV. Joyes Nostre Dame (ff. 47r-48r); Dit de la Pastoure (ff. 48r-62v); Oroyson de Nostre Seigneur (ff. 63r-65r) et Livre du duc des vrais amans (ff. 65r-98r).

Ce manuscrit possède une foliotation moderne en chiffres arabes. La reliure était anciennement en velours cramoisi sur bois, mais est à présent en peau couleur havane marbré, avec un entourage, vraisemblablement moderne, de palmettes dorées, le dos possède des nervures. On note l'absence de titre. Les tranches sont dorées.

Bibliographie: Catalogue des manuscrits français. Tome premier, ancien fonds, publié par ordre de l'Empereur, Paris, Librairie Firmin Didot, 1868, p. 9394 ; P. Paris, Les Manuscrits françois de la Bibliothèque du Roi, Paris, Techner, tome VI, 1845, p. 399-402; J. C. Laidlaw, «Christine de Pizan - A Publisher's Progress ", The Modern Language Review, 82 : 1, 1983, p. 35-75 ; J. C. Laidlaw, «Christine de Pizan - An Authors' Progress», The Modern Language Review, 78 : 3, 1987, p. 532-550. base).

- ms. Londres, British Library, Harley 4431 (ff. 267 r-v) : A2 (manuscript de

Manuscrit en vélin mesurant 380 x $280 \mathrm{~mm}$, composé de 398 feuillets, contenant 130 miniatures, et rédigé sur deux colonnes. Ce manuscrit a été donné par Christine de Pizan à la reine Isabeau de Bavière vers 1414. Il est communément appelé «Manuscrit de la Reine». Il renferme les textes suivants: Cent ballades (ff. 4r-21r); Virelais (ff. 21r-24r); Ballades d'estrange façon (ff. 24r-25r); Lais (ff. $25 \mathrm{r}-28 \mathrm{v}$ ); Rondeaux (ff. 28v-34r); Jeux a vendre (ff. 34v-51r); Autres ballades (ff. 37v-48r); Encore autres ballades (ff. 49v-51r); Complaintes amoureuses (ff. 48r-49v, 56v-58r); Epistre au dieu d'Amours (ff. 51r-56v); Débat de deux amans (ff. 58v-71r); Livre des trois jugemens (ff. 71v-81r); Dit de Poissy (ff. 81r94r) ; Epistre d'Othea (ff. 95r-141v); Livre du duc des vrais amans (ff. 143r-177v);

${ }^{36}$ Nous conservons les sigles adoptés par M. Roy pour son édition des Euvres poétiques de Christine de Pizan, op. cit., tome I, p. v-xxv.

${ }^{37}$ Voir J. Laidlaw, « Christine de Pizan - A Publisher's Progress », op. cit. 
Livre du chemin de long estude (ff. 178r-219v); Dit de la Pastoure (ff. 221r-236r); Epistres sur le Roman de la Rose (ff. 237r-254r) ; Epistre a Eustace Morel (ff. 255v257r); Oroyson Nostre Seigneur (ff. 257r-258r); Proverbes moraux (ff. 259v261v); Enseignemens moraux (ff. 261v-265v); Oroyson Nostre Dame (ff. 265r266v) ;.XV. Joyes Nostre dame (ff. 267r-v); Livre de Prudence (ff. 268r-287v); Livre de la cité des dames (ff. 288v-374r); Cent ballades d'amant et de dame (ff. 376r-398r).

On trouve sur le feuillet de garde une mention («Henry Duke of Newcastle, his booke, $1676 »)$ précisant que ce manuscrit a fait partie de la collection du duc de Newcastle au XVII ${ }^{\mathrm{e}}$ siècle. Ce manuscrit est recouvert de maroquin vert, et porte sur le dos un titre en anglais et sa cote en lettres dorées depuis 1962. On trouve également des ornements dorés sur les plats. Il est aujourd'hui composé de deux volumes.

Bibliographie: Catalogus Librorum Mss, Londres, s. d., p. 144; The Illuminator's Magazine, 1862, $\mathrm{n}^{\circ} 8$ et 9 ; S. Hindman, «The Composition of the Manuscript of Christine de Pizan's Collected Works in the British Library: A Reassassement », The British Library Journal, IX, 1983, p. 93-123 ; S. Hindman, «The Iconography of Queen Isabeau de Bavière (1410-1415): An Essay in Method », Gazette des Beaux-Arts, CII, 1983, p. 102-110 ; J. C. Laidlaw, "Christine de Pizan - A Publisher's Progress », The Modern Language Review, 82: 1, 1983, p. 35-75 ; J. C. Laidlaw, « Christine de Pizan - An Authors' Progress », The Modern Language Review, 78 : 3, 1987, p. 532-550 ; G. Ouy et Ch. Reno, «Les hésitations de Christine : études des variantes graphiques dans trois manuscrits autographes de Christine de Pizan », Revue des Langues Romanes, 92, 1988, p. 265-286; L. Schaefer, "Die Illustrationen zu den Handschriften der Christine de Pizan», Marburger Jahrbuch für Kunstwissenchaft, X, 1937, p. 119-208; W. Wells, « A Smile in Christine de Pizan for Christ's Conception », Journal of the Warburg and Courtland Institute, 2, 1938-1939, p. 68-69.

- ms. Paris, BnF. fr. 12779 (ff. 156 r-v) : B2.

Manuscrit (Mouchet suppl. fr. 6259) en papier mesurant 330 × $260 \mathrm{~mm}$, composé de 174 feuillets, écrit sur deux colonnes de 32 lignes. Il contient neuf miniatures en grisaille. Il renferme les textes suivants : Cent ballades (ff. 1r-21v); Virelais (ff. 21(bis)r-24v); Ballades d'estrange façon (ff. 24v-25r, 30v); Lais (ff. 37r-39r) ; Rondeaux (ff. 39r-45r); Jeux a vendre (ff. 45v-48v); Autres ballades (ff. 25r-32v); Complaintes amoureuses (ff. 33r-34v); Epistre au dieu d'Amours (ff. 65v-71v); Débat de deux amans (ff. 50r-65r); Dit de la Rose (ff. 72r-77r); Livre des trois jugemens (ff. 77v-89v); Dit de Poissy (ff. 30r-106r); Epistre d'Othea (ff. 106v-141r); Dit de la Pastoure (ff. 157r-174v); Epistres sur le Roman de la Rose (ff. 141v-149v); Enseignemens moraux (ff. 149v-153v); Oroyson Nostre Dame (ff. 154r-155v); .XV. Joyes Nostre Dame (ff. 156r-v).

Ce manuscrit, datant vraisemblablement de la seconde moitié du $X V^{\mathrm{e}}$ siècle, est défectueux de plusieurs feuillets. Il a appartenu au XVIII ${ }^{\mathrm{e}}$ siècle à Lacurne de Sainte-Palaye qui en fit faire deux copies: ms. Paris Bibliothèque de l'Arsenal 3295(B4) et Paris, BnF Moreau 1686.

On observe la présence de deux foliotations de deux mains différentes, toutes deux en chiffres romains. La première est contemporaine du manuscrit : du début au 
folio CVI inclus, la seconde: du folio CVII au folio CLXXIIII, qui ne tient pas compte de la lacune des folios CVI et CVII. La reliure est en peau couleur havane marbré, entourée sur les plats d'une frise florale dorée aux petits fers. Le dos est moderne, certainement refait, couleur havane, sans nerfs. Sur deux étiquettes de peau noire, on peut lire : «Collection Mouchet » et « Poésies de Christine de Pisan ».

Bibliographie: J. C. Laidlaw, "Christine de Pizan - A Publisher's Progress », The Modern Language Review, 82: 1, 1983, p. 35-75; G. Ouy et Ch. Reno, «Les hésitations de Christine : études des variantes graphiques dans trois manuscrits autographes de Christine de Pizan », Revue des Langues Romanes, 92, 1988, p. 265-286.

- ms. Chantilly, Musée Condé 492 (ff. 163 r-v) : B3.

Manuscrit (anc. 1667, Dupuy 466, Rigault 593) en vélin mesurant 290 x 242 mm et composé de 182 feuillets, écrit sur deux colonnes de 32 lignes. Il contient quatorze miniatures en grisaille. Ce manuscrit forme avec le ms. Chantilly, Musée Condé 493, un recueil des Euvres de Christine de Pizan, dont il forme la première partie. Ces deux volumes constituent une collection qui, peut-être, aurait été préparée à l'attention de l'épouse du duc Louis d'Orléans, Valentine Visconti. Le manuscrit est daté, on en connaît donc sa date d'achèvement: 23 juin 1402. Il renferme les textes suivants: Cent ballades (ff. 2r-22v); Virelais (ff. 23r-26v); Ballades d'estrange façon (ff. 26v-27v, 32v) ; Lais (ff. 37r-47r) ; Rondeaux (ff. 41r47r); Jeux a vendre (ff. 47v-51r); Autres ballades (ff. 27r-34v); Complaintes amoureuses (ff. 35r-36v); Epistre au dieu d'Amours (ff. 67v-73v); Débat de deux amans (ff. 51v-67r); Dit de la Rose (ff. 74r-79r) ; Livre des trois jugemens (ff. 79v91v); Dit de Poissy (ff. 92r-108r); Epistre d'Othea (ff. 108v-148r); Livre du chemin de long estude (ff. 184r-131v); Dit de la Pastoure (ff. 166r-182v); Epistres sur le Roman de la Rose (ff. 148v-156r); Oroyson Nostre Seigneur (ff. 164r-165v); Enseignemens moraux (ff. 156v-160v); Oroyson Nostre Dame (ff. 161r-162v);.XV. Joyes Nostre dame (ff. 163r-v); Livre de la cité des dames (ff. 1r-30r).

Ce manuscrit, qui appartenait au comte de Toustain, a été déposé par ce dernier chez les libraires Morgand \& Fatout en 1882. Le duc d'Aumale l'a acquis en 1887. Il est entré dans les collections du Musée Condé en 1888. Il est recouvert d'une riche reliure en maroquin rouge signée «Belz-Niedrée ».

Bibliographie : Répertoire général de la Librairie Morgand et Fatout, Paris, 1882, p. 190, $\mathrm{n}^{\circ} 1482$; Chantilly. Le cabinet des livres manuscrits, Paris, Librairie Plon, vol. II, 1900, p. 84-86; J. C. Laidlaw, «Christine de Pizan - A Publisher's Progress ", The Modern Language Review, 82: 1, 1983, p. 35-75; G. Ouy et Ch. Reno, «Les hésitations de Christine : études des variantes graphiques dans trois manuscrits autographes de Christine de Pizan », Revue des Langues Romanes, 92, 1988, p. 265-286.

- ms. Paris, Arsenal 3295 (ff. 307v-308v) : B4.

Manuscrit sur papier mesurant 380 x $250 \mathrm{~mm}$, composé de 344 feuillets, ne contenant aucune miniature, et rédigé sur une colonne. Ce manuscrit, dont l'écriture est vraisemblablement du XVIII ${ }^{\mathrm{e}}$ siècle, est une copie du ms. Paris, BnF fr. 12779 (B2). Il renferme les textes suivants: Cent ballades (ff. $3 \mathrm{r}-44 \mathrm{v})$; Virelais (ff. 45r52r) ; Ballades d'estrange façon (ff. 52r-53v, 64r-v); Lais (ff. 73r-77r) ; Rondeaux 
(ff. 77v-89v); Jeux a vendre (ff. 90r-96v); Autres ballades (ff. 53v-68v); Complaintes amoureuses (ff. 69r-72v); Epistre au dieu d'Amours (ff. 128v-140v); Débat de deux amans (ff. 97r-127v); Dit de la Rose (ff. 141r-151r); Livre des trois jugemens (ff. 151v-175v); Dit de Poissy (ff. 176r-208v); Epistre d'Othea (ff. 209r278v); Dit de la Pastoure (ff. 309r-344v); Epistres sur le Roman de la Rose (ff. 279r-294r); Enseignemens moraux (ff. 294v-303r); Oroyson Nostre Dame (ff. 303v-307r) ; .XV. Joyes Nostre Dame (ff. 307v-308v).

Ce manuscrit a été la propriété de Lacurne de Sainte-Palaye. Il est recouvert d'une reliure en basane.

Bibliographie: H. Martin, Catalogue des manuscrits de la Bibliothèque de l'Arsenal, Paris, Librairie Plon, 1887, p. 311.

\section{Établissement du texte}

Nous avons, pour établir l'édition des .XV. Joyes nostre Dame rimees, fidèlement transcrit le texte du manuscrit Londres, British Library, Harley 4431 pour deux raisons, déterminantes à nos yeux : les recherches menées ces dernières années ont clairement mis en évidence que ce manuscrit est autographe d'une part ${ }^{38}$; Christine de Pizan semble en avoir supervisé l'élaboration d'autre part ${ }^{39}$.

Nous avons résolu les abréviations-peu nombreuses- lorsqu'il était nécessaire de le faire, et les avons transcrites selon les occurrences présentes dans le texte. Nous avons suivi les conventions d'édition établies par Mario Roques ${ }^{40}$ ainsi que celles élaborées par Alfred Foulet et Mary B. Spear ${ }^{41}$. Nous avons également systématiquement distingué $\mathrm{i} / \mathrm{j}$ et $\mathrm{u} / \mathrm{v}$. Nous sommes responsables de la ponctuation.

\footnotetext{
${ }^{38}$ Voir G. Ouy et Ch. Reno, «Identification des manuscrits autographes de Christine de Pizan », Scriptorium, 34, 1980, p. 221-238; G. Ouy, «Une énigme codicologique : les signatures des cahiers de Christine de Pizan», in Calames et cahiers: mélanges de codicologie et de paléographie offerts à Léon Gilissen, éd. J. Lemaire et E. van Balberghe, Bruxelles, Centre d'Études des Manuscrits, 1985, p. 119-131; G. Ouy et Ch. Reno, «Les hésitations de Christine : études des variantes de graphies dans trois manuscrits autographes de Christine de Pizan ", Revue des Langues Romanes, 92, 1988, p. 265-286 et Ch. C. Willard, "An autograph manuscript of Christine de Pizan? », Studi Francesi, 27, 1965, p. 452-457 qui, la première, mit en évidence que L'Epistre a la Reine conservée dans le manuscrit BnF fr. 580 aurait été copiée par Christine elle-même.

${ }^{39}$ Voir S. Hindman, Christine de Pizan's « Epistre Othea » : Painting and Politics at the court of Charles VI, Toronto, Pontifical Institut of Mediaeval Studies, 1986, qui montre bien que Christine intervient dans l'exécution des enluminures.

40 M. Roques, "Règles pour l'édition des anciens textes français et provençaux", Bibliothèque de l'École des Chartes, 87, 1926, p. 453-459 - Rééd.: Romania, 52, 1926 , p. 243-249. Nous avons également consulté P. Meyer, «Instruction pour la publication des anciens textes », Bulletin de la Société des Anciens Textes Français, 35, 1909, p. 64-79Rééd. : Bibliothèque de l'École des Chartes, 71, 1910, p. 224-233; J. Acher, « De l'emploi du tréma ", Revue des Langues Romanes, 56, 1913, p. 458-465 et Conseils pour l'édition des textes médiévaux, Fascicules I, II et III, Paris, Comité des Travaux Historiques et Scientifiques-École Nationale des Chartes, 2002.

${ }^{41}$ A. Foulet et M. B. Spear, On editing Old French Texts, Lawrence, Regets, 1979.
} 
Enfin, le texte présentant une erreur (strophe X, vers 43), nous avons procédé à sa correction, que nous signalons dans le corps du texte entre crochets.

Nous avons disposé notre apparat critique comme il est convenu de le faire, c'est-à-dire sur deux étages. Le premier comporte les leçons rejetées et le second les variantes des autres manuscrits. 


\section{Les .XV. Joyes Nostre Dame rimees, ms. Londres, British Library, Harley 4431}

Ci commencent les .XV. Joyes Nostre Dame rimees. (f. $267 \mathrm{r}$ a)

Glorieuse Dame, je te salue

Trés humblement, d'icelles .XV. joyes

Qu'en terre eus, doulce dame impolue, Par ce te pry que reconfort m'envoyes.

Ave Maria.

\section{I}

$5 \quad$ Priez pour moy, beneuree pucelle, Pour le salu qui vint de Dieu le pere Que te porta l'archange et la nouvelle Que du filz Dieu seroies vierge mere.

Ave Maria.

\section{II}

Empetres moy grace pour celle joye Que tu eus quant ta cousine encontras Elisabeth, qui salu en la voye Te donna lors qu'en sa maison entras.

$$
\text { Ave Maria }
$$

III

Doulce Dame, vueilles pitié avoir De mon ame pour ycelle leece Que tu eus quant en ton ventre mouvoir Le filz de Dieu sentis plain de sagece.

$$
\text { Ave Maria. }
$$

Rubrique Cy commencent les .XV. Joyes Nostre Dame. Al; Cy commencent les .XV. Joyes de Nostre Dame rimees. B2B3B4.

2- h. de celles .XV. A1.

3- e. teur e. B3.

I-5 m. doulce dame p. B2 B3 B4.

I-6 Par 1. B2 B3 B4.

I-7 q. t'aporta 1. A1; q. t'apporta 1. B2 B3 B4.

I-8 f. de D. $B 2$; v. et m. $B 3$.

II-12 1. que e. B2 B3 B4. 


\section{IV}

Digne Vierge, qui le sauveur portas, Pour ycelle grant joye, secourir Tu me vueilles, que eus quant l'enfantas Et vien a moy quant je devray mourir.

$$
\text { Ave Maria }
$$

V

O Marie, gardes moy des faulx tours Et de l'agait de l'ennemi soubtil, Pour la joye que eus quant les pastours Te trouverent et ton benoit chier fil.

Ave Maria.

VI

25 Pour la grant joye et consolacion,

Doulce Dame, que eus, quant les .IIJ. roys

A ton doulx filz par grant devocion

Vindrent offrir, gard moy de tous desrois.

Ave Maria.

VII

Pour ycelle joye que eus, chere Dame,

30 Quant ton cher filz trés digne offris au temple, De mal garder vueilles mon corps et m'ame Et me donnes vivre a ton bon exemple.

Ave Maria

IV-20 E. bien amoy q. $B 4$.

VII-29 j. qu'e. $B 2 B 3 B 4$.

VII-30 o. ou t. B2 B3 B4. 


\section{VIII}

Reconfortes mon las cuer esperdu Pour la joye que eus quant retrouvas

35 Ton chier enfant que avoies perdu, Et au Temple, Dame, tu le trouvas.

Ave Maria.

\section{IX}

Vierge, pries qu'a moy aydier enclin Soit ton doulx filz, pour ycelle grant joye Qu'eus aux nopces de saint Archedeclin, Quant d'iaue vin fist par divine voye.

$$
\text { Ave Maria. }
$$

$\mathrm{X}$

Que mon corps gard de toutes meffaçons Pries ton filz, pour la joye sacree Que eus quant de .V. pains d'orge et [de .IJ.] poissons Cinq mille hommes replanis en la pree.

$$
\text { Ave Maria. }
$$

XI

45 Pour la joye qui en toy abita, Trés doulce Dame, en ycelle journee Que ton doulx filz de mort ressuscita, Me soit joye celestiel donnee.

Ave Maria.

IX-39 s. Archetheclin A1.

X-43 e. .IIJ. p. corr. d'après $A 1 B 2 B 3 B 4$.

X-43 Qu'e. B3.

$\mathrm{X}-44$ h. replein e. $B 4$.

XI-46 d. $a$ y. $B 2 B 3 B 4$. 


\section{XII}

Doulce Dame, pour la joye plainiere

Qu'a Pasques eus quant ton filz t'apparu (f. $267 \mathrm{v}$ a)

Ressuscité, exausses ma priere,

Et mets en moy la mort dont il mouru.

Ave Maria.

XIII

Pries ton filz qu'aprés lui il me traye,

Pour la joye que eus a l'Assancion,

55 Quant il monta ou ciel, c'est chose vraye,

Si vrayement ay je salvacion.

Ave Maria.

XIV

Doulce Dame, pour ycellui plaisir Que tu eus quant le Saint Esperit vint Aux appostres conforter leur desir,

60 Ainsi me puist venir com leur advint.

Ave Maria.

XV

Vierge digne, pour la joye enterine

Que eus au jour de ton Assompcion Qu'en ciel montas, de pechié la racine Ostes de moy et mets devocion.

Ave Maria.

Explicit.

XIII-54 j. qu' e $B 2 B 3 B 4$.

XIII-55 m. $a u$ c. $B 4$.

XIII-56 v. aye s. B3 ; a. je s. omis B2 B3 B4; a. salutacion B4.

$\mathrm{XV}-62 Q u$ 'e. $B 3$.

XV-63 Que ou c. B2 B3 B4.

Ave Maria omis B2 B3 B4.

Explicit le Livre de Christine B2 B4; Explicit omis B3 ; Amen B2 B3 B4; Deo Gracias B2 B4. 


\section{Notes}

Le nombre romain renvoie au numéro de la strophe, le nombre arabe renvoie, quant à lui, au numéro de vers.

Rubrique - Le manuscrit $A 2$ donne $C i$ commencent les .XV. Joyes nostre Dame rimees, tandis que l'on trouve Cy commencent les .XV. Joyes nostre Dame dans $A 1$ et $C y$ commencent les .XV. Joyes de nostre Dame dans B2, B3 et B4.

Si Christine traite des quinze joies de la Vierge, notons que c'est au bas Moyen Âge que la spiritualité fixa une liste des «Sept joies» et des «Sept douleurs » ressenties par Marie durant sa vie :

\begin{tabular}{|l|l|}
\hline Sept joies & Sept douleurs \\
\hline Annonciation & Prophétie de Syméon \\
\hline Visitation & Fuite en Egypte \\
\hline Naissance du Christ & Jésus perdu au Temple \\
\hline Adoration des Mages & Passion du Christ \\
\hline Rencontre de Syméon & Crucifixion \\
\hline Jésus retrouvé au Temple & Descente de Croix \\
\hline Couronnement céleste & Inhumation \\
\hline
\end{tabular}

Voir l'entrée «Marie», in Dictionnaire illustré de la Bible, Paris, Bordas, 1990, p. 401-401, (p. 401-402).

3 - Le manuscrit $B 3$ donne teur pour terre.

I - L'Annonciation : (Lc 1, 26-38)

Le texte des.$X V$. Joyes trouve sa correspondance à la strophe III de l'Oroyson Nostre Seigneur:

«Fay mon cueur lié en toy pour le salu

Qu'a la Vierge par Gabriel tramis

Lui annonçant que pour nostre salu

T'enfanteroit comme il estoit promis. »

(v. 9-12, ms. Londres, British Library, Harley 4431, f. 257r)

La fête de l'Annonciation est célébrée le 25 mars.

I-7: Bien que tous les manuscrits donnent q. t'apporta 1., nous ne corrigeons pas, car ni le sens du texte ni la métrique du vers ne sont altérés dans le manuscrit $A 2$.

II - La Visitation: (Lc 1, 39-56). La Visitation (Visitatio Beatae Maria Virginis en latin) désigne la visite qu'effectua Marie auprès d'Elisabeth. Voir 
Dictionnaire illustré de la Bible, op.cit., p. 582. La fête de la Visitation de Marie est célébrée le 31 mai. Elisabeth était la cousine de Marie, et la femme d'un prêtre de village appelé Zacharie. Voir l'entrée "Elisabeth", in R. Brownrigg, Les Personnages du Nouveau Testament, Paris, Compagnie Française de Librairie, 1979, p. 97-99 et Dictionnaire des noms propres de la Bible, Paris, Le Cerf, 1996, p. 123.

III - Évocation des premiers tressaillements de l'enfant : (Lc 1, 41).

IV - La Nativité : (Lc 2, 6-7).

Le texte des.$X V$. Joyes trouve sa correspondance à la strophe IV de l'Oroyson Nostre Seigneur:

«O bon Jhesus, pour ta Nativité

Conforte moy et ayde en tout besoing,

De mes pechiez oste l'iniquité

Et ne seuffres que de toye soye loing. »

(v. 13-16, ms. Londres, British Library, Harley 4431, f. 257r)

Sur les récits de la Nativité, voir l'entrée "Jésus», in R. Brownrigg, Les Personnages du Nouveau Testament, op. cit., p. 207-243, (p. 212-217).

IV - 20 : Le manuscrit $B 4$ donne E. bien a., que nous ne notons pas comme variante, car il s'agit manifestement d'une faute de copiste qui n'a pas de sens.

V - Visite des bergers : (Lc 2, 8-16).

Le texte des . $X V$. Joyes trouve sa correspondance à la stophe $\mathrm{V}$ de l'Oroyson Nostre Seigneur :

«Trés doulx enfant, Emanuel plaisant,

Je te requier que pour yceulz pastours

Qui en creche te trouverent gisant,

En tous besoings j'aye de toy secours. »

(v. 17-20, ms. Londres, British Library, Harley 4431, f. 257r-v)

L'image du berger est à la fois récurrente et importante aussi bien dans l'Ancien Testament dans dans le Nouveau Testament. Cette image peut être appliquée aux rois, à Dieu, à Jésus ou bien encore aux Ministères, voir Dictionnaire Illustré de la Bible, op. cit., p. 106-107 et Dictionnaire encyclopédique de la Bible, Paris, Brepols, 1987, p. 198-199.

VI - Oblation des mages : (Mt 2, 1-2).

Le texte des.$X V$. Joyes trouve sa correspondance à la strophe VII de l'Oroyson Nostre Seigneur:

« Ottroye moy toy faire oblacion

Et offrande qui te soit agreable,

Si com firent par grant devocion 
Les bons .iij. roys que tu os acceptable. »

(v. 25-28, ms. Londres, British Library, Harley 4431, f. 257v)

Le terme de mage est utilisé dans la tradition pour désigner les étrangers venus adorer l'enfant Jésus, voir Dictionnaire encyclopédique de la Bible, op. cit., p. 767 et Dictionnaire illustré de la Bible, op. cit., p. 391-392. Voir également: A. Carnoy, «Le nom des mages », Le Muséon, 27, 1908, p. 121-158 J. C. MarschEdwards, «The Magi in Tradition and Art», Irish Ecclesiastical Record, 85, 1956, p. 1-19.

VII - L'Offrande au Temple : (Lc 2, 22).

Le texte des.$X V$. Joyes trouve sa correspondance à la strophe VIII de l'Oroyson Nostre Seigneur:

«L'offrande que de toy ta mere au Temple

Fist, doulx Jhesus, es bras du saint prophete

Symeon qui t'atendoit, soit exemple

De m'ame offrir a toy Dieux qui l'as faicte. »

(v. 29-32, ms. Londres, British Library, Harley 4431, f. 257v)

VIII - Jésus retrouvé au Temple : (Lc 2, 41-51).

IX - Noces de Cana : (Jn 2, 1-11).

Cana est une localité de Galilée. Sa localisation est incertaine. «On a voulu la situer à Kefar Kanna à $6 \mathrm{~km}$ au nord de Nazareth, mais il faut probablement la placer à 14,5 km au nord de Nazareth à hirbet Qana dont l'environnement marécageux justifierait bien son nom (gr. kana, de l'hébreu qanah: «roseau») : Dictionnaire encyclopédique de la Bible, op. cit., p. 227. Voir Dictionnaire des noms propres de la Bible, op. cit., p. 86 et Dictionnaire illustré de la Bible, op. cit., p. 119-120.

IX-39: Le manuscrit $A 1$ est le seul à transcrire Archetheclin pour Archedeclin. Archedeclin ou Arcedeclin ou Architriclin désigne l'époux des noces de Cana (Raoul de Cambrai), et un organisateur de festin (Villon, Grand Testament). Dans l'Antiquité ce terme désignait celui qui présidait à l'ordonnance d'un festin. Familièrement, il est celui qui organise un repas. Il est le saint patron des maîtres d'hôtel.

$\mathbf{X}$ - Allusion au Miracle des 5 pains : (Lc 9, 13-17), (Jn 6, 9-11).

X-43: Il s'agit de la seule faute commise par le manuscrit $A 2$. L'erreur n'est pas très grave, mais il convient toutefois de corriger le texte afin d'être en accord avec les Evangiles qui parlent bien de deux poissons et non de trois.

XI - Résurrection : (Lc 24, 1-53). Voir également (Mt 28, 1-20); (Mc 16, 118) et (Jn 20, 1-21, 23). 
La résurrection de Jésus signifie qu'il vit au-delà de la mort, voir Dictionnaire encyclopédique de la Bible, op. cit., p. 1110-1113; Dictionnaire Illustré de la Bible, op. cit., p. 515-516; l'entrée "Jésus», in R. Browrigg, Les Personnages du Nouveau Testament, op. cit., p. 207-243, (p. 236-238) ; J. Comblin, La Résurrection de Jésus-Christ, Paris, éditions universitaires, 1959; J. Daniélou, La Résurrection, Paris, Le Seuil, 1969; F. X. Durwell, La Résurrection de Jésus, mystère de salut, Paris, éditions du Cerf, 1976; G. Deluz, La Résurrection de Jésus : croire et comprendre, Genève, Labor et Fides, (Essais Bibliques, 33), 2003; X. Léon-Dufour, Résurrection de Jésus et message pascal, Paris, Le Seuil, 1971; W. Marxsen, Die Auferstehung Jesus von Nazareth, Gütersloh, Gütersloher Verlag G. Mohn, 1968 et A. E. Mussard, La Résurrection de Jésus-Christ : étude historique et critique, Genève, Imprimerie Gilbert et Cie, 1910.

XII - Apparition du ressuscité : (Mt 28, 1-20), (Mc 16, 1-18), (Jn 20, 1-29; 21,1-23).

Le texte des.$X V$. Joyes trouve sa correspondance à la strophe LVII de l'Oroyson Nostre Seigneur:

«Confortes moy en mon adversité

Si com tu feis ta mere, a qui en joye

Tu t'apparus en corps resuscité,

Et aussi a Magdelaine en la voye. »

(v. 225-228, ms. Londres, British Library, Harley 4431, f. 259r)

XIII - L’Ascension : (Lc 24, 51), (Ac 1, 9-10)

Le texte des.$X V$. Joyes trouve sa correspondance à la strophe LIX de l'Oroyson Nostre Seigneur:

«Mon doulx sauveur, pour celle Assencïon

Ou tu montas ou ciel, presens les tiens,

Quarente jours puis ta surrexïon,

M'ame et mon corps, en toy servant, maintiens. »

(v. 233-236, ms. Londres, British Library, Harley 4431, f. 259r)

L'Ascension désigne la montée du Christ au ciel sous les yeux des Apôtres, voir Dictionnaire encyclopédique de la Bible, op. cit., p. 149-150; Dictionnaire illustré de la Bible, op. cit., p. 78-81; l'entrée «Jésus", in R. Brownrigg, Les Personnages $d u$ Nouveau testament, op. cit., p. 207-243, (p. 238-239); V. Larrañaga, L'Ascension de Notre Seigneur dans le Nouveau Testament, traduit de l'espagnol par G. Cazaux, Rome, Institut Biblique Pontifical, 1938; P. Benoit, «L'Ascension», Revue Biblique, 56, 1949, p. 161-203; G. Schille, «Die Himmelfahrt », Zeitschrift für die Neutestamentliche Wissenchaft und die Kunde des Alten Christentums, 57, 1966, p. 183-199; S. G. Wilson, «The Ascension. A Critique and an Interpretation ", Zeitschrift für die Neutestamentliche Wissenchaft und die Kunde des Alten Christentums, 59, 1968, p. 269-281 et C. M. Martini, «L'Ascension de Jésus », Assemblées du Seigneur, 28, 1969, p. 6-11.

La fête de l'Ascension est célébrée le 29 mai. 
XIV - La Pentecôte : (Ac 2, 1-4).

Le texte des .XV. Joyes trouve sa correspondance à la strophe LX de l'Oroyson Nostre Seigneur:

«Beau Sire Dieux, et si qu'a Penthecouste

Enluminas tes appostres du Saint

Esperit, vueilles qu'icelle doulceur gouste

Qu'ilz sentirent que ta vertu ençaint. »

(v. 237-240, ms. Londres, British Library, Harley 4431, f. 259r)

La Pentecôte est considérée comme la fête de l'envoi du Saint Esprit et de la fondation de l'Église, voir Dictionnaire Encyclopédique de la Bible, op. cit., p. 1003-1004 et Dictionnaire illustré de la Bible, op. cit., p. 477.

La fête de la Pentecôte est célébrée le 8 juin.

XV - L'Assomption.

L'Assomption (Assumptio Beatae Mariae Virginis en latin) désigne l'élévation au ciel de Marie. Elle n'est pas mentionnée dans le Nouveau Testament et n'apparaît que tardivement dans les apocryphes, voir Dictionnaire illustré de la Bible, op. cit., p. 82-85.

La fête de l'Assomption est célébrée le 15 août.

XV - Tous les manuscrits de la famille B (B2, B3 et B4) omettent Ave Maria à la fin de cette prière qu'ils remplacent par Amen. Notons que dans B2 et B4 nous trouvons les formules Explicit le Livre de Cristine, Deo Gracias, cet explicit se rapportant dans les deux cas à tout le manuscrit. $B 3$ ne s'achève que par Amen et $A 2$ et $A 1$ se terminent par Explicit. 


\section{Glossaire}

Le nombre romain renvoie au numéro de la strophe, le nombre arabe renvoie, quant à lui, au numéro de vers.

agait: n.m. V, 22, embuscade.

aydier : $v$. IX, 37, aider.

beneuree : adj. I, 5, bienheureuse.

benoit : adj. V, 24, béni.

celestiel : adj. XI, 48, céleste.

conforter : v. XIV, 59, réconforter

consolacion : $n . f$. VI, 25 , réconfort, soulagement.

desrois : n.m. VI, 28, déroute.

empetrer : $v$. II, 9, impétrer, faire une demande, requérir, supplier.

enclin : adj. IX, 37, incliné.

encontrer : $v$. II, 10, rencontrer.

enterine : adj. XV, 61, complète, sincère, pure.

esperdre : $v$. VIII, 33, troubler, égarer.

glorieuse : adj. 1, qui mérite la gloire.

impolue : adj. 3, pure.

leece : $n . f$. III, 14 , joie.

meffaçons : $n . f . \mathrm{X}, 41$, erreurs.

mouvoir : $v$. III, 15 , bouger.

pastour: n. m. V, 23, berger.

plainiere : adj. XII, 49, grande, importante.

pree : $n . f . \mathrm{X}, 44$, pré, prairie.

racine : $n . f . \mathrm{XV}, 63$, racine, souche.

reconfort: $n . m .4$, secours.

replanir : $v$. X, 44, combler (de satisfaction).

sagece : $n . f$. III, 16 , circonspection.

salu : $n . f . \& m$. I, 6, salut éternel ; II, 11, action de saluer.

salvacion : $n . f$. XIII, 56, salut.

soubtil : adj. $\mathrm{V}, 22$, perfide.

tour: $n . m$. V, 21 , ruse.

traire : $v$. XIII, 53, tirer.

voye : $n . f$. IX, 40, manière, façon.

Jean-François Kosta-Théfaine

Centre d'Études des Textes Médiévaux - Rennes 2 